\title{
Ageing-induced changes in the cortical granules of mouse eggs
}

\author{
Hugo Díaz and Pedro Esponda* \\ Centro de Investigaciones Biológicas, CSIC, Madrid, Spain
}

Date submitted: 11.9.03. Date accepted: 22.12.03

\section{Summary}

The cortical cytoplasm and cortical granules (CGs) of mouse oocytes were analysed by electron microscopy. Oocytes were collected soon and $20 \mathrm{~h}$ after ovulation from adult young females (3-4 months old). In addition, gametes collected soon after ovulation from 12- to 14-month-old females were used. Ultrastructural analyses were undertaken using the conventional procedures and the alcoholic PTA method. PTA selectively stains the CGs indicating the presence of lysine-rich proteins in these granules. Oocytes from young females showed CGs as dense granules $300-500 \mathrm{~nm}$ in diameter linearly arranged under the oolemma. In oocytes recovered $20 \mathrm{~h}$ after ovulation $24.31 \%$ of CGs appeared vacuolated and $38.40 \%$ internalized in the cytoplasm. In gametes collected from old females several changes were observed in the cortical cytoplasm: (a) CGs appeared concentrated in some areas while others regions were devoid of granules; (b) groups of CGs appeared internalized in the egg cytoplasm; (c) the CG contents had swollen and changed, showing dense and clear areas; (d) numerous dense structures and vesicles (lysosome-like vesicles) were present; (e) cytoplasmic fragmentation was frequently seen. Fragments contained CGs, dense structures and vacuoles. These changes are closely related to the low fertilization rates shown by these oocytes when they were used for in vitro fertilization procedures.

Keywords: Ageing, Cortical granules, Electron microscopy, Oocyte, In vitro fertilization

\section{Introduction}

The fertile life of the mammalian female is interrupted when hormonal changes occur and the ovulation cycle ends. In the female mouse the duration of the fertile life depends on the strain, but usually at 8-10 months of age a decrease in fertility occurs (Adams, 1984). In rodents, in contrast to man, oocytes are not totally depleted at the time of menopause and ovulation does not cease, but there is a significant decrease in the number of ovulated oocytes depending on the age (Adams, 1984). On the other hand, the fertile life of the mammalian ovulated oocyte is short and it has been shown that after a few hours in the oviduct numerous changes occur in the structure and physiology of the gamete (Szollosi, 1971; Gulyas, 1980; Ducibella, 1996; Xu et al., 1997; Tarín et al., 2000). On the other hand, oocytes recovered from old females also show a series of changes in different

\footnotetext{
*All correspondence to: Dr P. Esponda, Centro de Investigaciones Biológicas, CSIC, Ramiro de Maeztu 9, 28040 Madrid, Spain. Tel: +34918373112. Fax: +34915630462. e-mail: esponda@cib.csic.es
}

nuclear and cytoplasmic structures (Peluso et al., 1980; Tarín et al., 2001).

Cortical granules (CGs) are a characteristic of the mammalian mature oocyte cytoplasm (Longo, 1985; Gulyas, 1980; Guraya, 1982). CGs are a Golgi product and in the mature oocyte they appear as small granules linearly arranged under the oolemma. CGs release their content by exocytosis upon fertilization due to the elevated calcium level that occurs at this time (Yanagimachi, 1988; Hoodbhoy \& Talbot, 1994). By their exocytosis CGs discharge their proteolytic contents into the perivitelline space, and induce changes in the zona pellucida that prevent the entry of additional sperms (Gulyas, 1980; Hoodbhoy \& Talbot, 1994). Despite numerous analyses of the changes that occur in different cell structures during oocyte ageing (Tarín et al., 2000, 2001), studies of CGs have rarely been carried out. In the mouse postovulatory ageing induces a migration of CGs to the inner region of the cytoplasm and its structure change, and it was claimed that the number of CGs is inversely proportional to the age of the ovum (Szollosi, 1971). A similar situation occurs in the human, in which a centripetal migration occurs 
in oocytes aged in culture (Sathananthan, 1997). On the other hand, in the rabbit it has been shown that postovulatory ageing in the oviduct induces an increase in CGs along the cortex (Longo, 1974), a phenomenon that also occurs in the hamster (Yanagimachi \& Chang, 1961).

The effects of preovulatory ageing on oocytes have not been so extensively studied as the consequences of postovulatory ageing (Tarín et al., 2000), and analyses devoted to the study of CGs are very scarce (Peluso et al., 1980). In oocytes recovered from old female mice, changes have been described as a decrease in the number of CGs, modifications in the zona pellucida intracellular mitochondrial aggregates, changes in the chromosomal characteristics, cellular fragmentation, etc. (Peluso et al., 1980; Tarín et al., 2001).

In the present report we show that remarkable changes occur in the CGs and in the egg cortex of mouse oocytes collected late after ovulation and in those ovulated by old females, and that these changes would have a role in the decrease in the fertilization rates observed.

\section{Materials and methods}

Adult female mice from the hybrid strain C57BL $\times$ CBA were used. Two groups of females were used: one comprised females 3-5 months old (young females) and other females 12-14 months old (old females). Animals were kept in the Animal Service of CIB (CSIC) in stable conditions of temperature and light (lights on at 07:00 hours and off at 19:00 hours) and with food and water ad libitum. Females were induced to ovulate using 5IU PMSG $12 \mathrm{~h}$ after an injection of 5 IU hCG. Three types of oocytes were employed: (a) oocytes recovered from adult young females 3-10 h after ovulation; (b) oocytes collected from young females $20 \mathrm{~h}$ after ovulation; (c) oocytes recovered from old females $3-10 \mathrm{~h}$ after ovulation. Gametes were collected in phosphate-buffered saline (PBS) medium at $\mathrm{pH}$ 7.3. In all cases females were killed using ether, and the animal protocol used was in accordance with the European Union Agreement about Vertebrate Animal protection (3/18/1986) and in accordance with the NIH guide and the CSIC (Spain) ethics committee for the care and use of laboratory animals.

\section{In vitro fertilization}

Egg collection was carried out as described above. Animals were anaesthetized using an intraperitoneal injection of Rompun and Ketolar (Parke-Davis, Madrid, Spain). Four milligrams of Ketolar and $0.35 \mathrm{mg}$ of Rompun in $1 \mathrm{ml}$ of PBS were used, and injected at a dosage of $21 \mu \mathrm{l} / \mathrm{g}$ body weight. Sperm were collected from the caudae epididymis of 3- to 4month-old males. Caudae were punctured in $200 \mu \mathrm{l}$ of pre-warmed capacitation medium (M16 medium supplemented with $40 \mathrm{mg} / \mathrm{ml}$ of bovine serum albumin and antibiotics). All reagents were purchased from Sigma (St Louis, MO, USA) and were embryo tested.

The sperm suspension was collected in a sterile tube and the sperm concentration estimated using a haemocytometer. Sperm were diluted in capacitation medium to give a final concentration of $1-2 \times 10^{6}$ cells / $\mathrm{ml}$. Capacitation took place for $3 \mathrm{~h}$ at $37^{\circ} \mathrm{C}$ in an atmosphere of $\mathrm{CO}_{2}$. At the end of incubation, $100 \mu \mathrm{l}$ drops from the sperm suspension were placed under mineral oil and the eggs were transferred to these drops and left overnight. At different time periods the inseminated eggs were examined for signs of nuclear decondensation and cleavage using a fluorescence and/or phase-contrast microscope. An antifading solution containing Vectashield (Vector Laboratories, Burlingame, CA, USA) and DAPI (Roche Diagnostics, Barcelona, Spain) as a DNA fluorescent stain was employed to analyse eggs and embryos.

\section{Electron microscopy}

Analyses were conducted using two procedures:

(a) Conventional method. Oocytes were fixed in a mixture of $2 \%$ glutaraldehyde, $1 \%$ paraformaldehyde and $0.1 \mathrm{M}$ sucrose in $0.5 \mathrm{M}$ cacodylate buffer ( $\mathrm{pH} 7.3$ ). Samples were thoroughly washed with the buffer and postfixed in $2 \%$ osmium tetroxide in cacodylate buffer. Then they were dehydrated in alcohol and embedded in an epoxy resin. Semithin sections were stained with $0.2 \%$ toluidine blue, and then they were observed under a light microscope in order to identify the appropriate oocytes. Ultrathin sections cut in a LKB Ultratome, and stained with uranyl acetate and lead citrate, were studied in a Philips 300 electron microscope.

(b) Alcoholic PTA staining. The method described by Sheridan \& Barnett (1969) was applied. Samples were fixed exclusively in $2 \%$ glutaraldehyde solution (in cacodylate buffer $\mathrm{pH} 7.3$ ) for $2 \mathrm{~h}$. Samples were thoroughly washed in the buffer and dehydrated in alcohols. Then they were immersed overnight in a solution of $1 \%$ phosphotungstic acid (PTA) in absolute ethanol, washed, dehydrated and embedded in Epon.

Thick sections $(2-5 \mu \mathrm{m})$ from (a) and (b) were cut with the Ultratome. These sections were stained using toluidine blue solution $(0.1 \%$ in borate buffer $)$ and observed under light or phase-contrast microscopy. Ultrathin sections from the PTA-treated material were analysed without further staining. In some cases semithin sections $(0.5-1 \mu \mathrm{m})$ from the PTAtreated material were observed under the electron microscope at $100 \mathrm{kV}$. A Philips 300 electron microscope 
was employed for observations. Selected electron micrographs from the PTA-treated material were used to count the CGs. In the case of young females, 14 freshly ovulated eggs and 12 eggs recovered $20 \mathrm{~h}$ after ovulation were analysed. In the case of old females we selected those eggs that showed morphological changes (an irregular surface and the presence of cytoplasmic extrusions) when observed under phasecontrast microscopy. In this case, out of 13 eggs we founded 9 that showed modifications and were analysed. In all cases various areas of the egg cortex were randomly selected and a total of 20-45 CGs were counted per egg and their structural characteristics recorded. The position of the CGs was also recorded, and classified as normal if they were close to the oolemma or internalized if they were located $2000 \mathrm{~nm}$ from the plasma membrane.

\section{Results}

\section{In vitro fertilization}

Of the oocytes collected from young females soon after ovulation, $66.93 \%(n=127)$ of them attained the 2-cell embryo stage. This percentage was reduced to $16.85 \%(n=89)$ when oocytes were collected $20 \mathrm{~h}$ after ovulation, and to $24.32 \%(n=128)$ in oocytes collected from old females. The microscopic analysis of these non-fertilized oocytes showed that no sperm penetration occurs, that spermatozoa remained attached to the zona pellucida or appeared in the perivitelline space, and that no pronuclear formation occurred.

Oocytes collected soon after ovulation from young females showed numerous microvilli, mitochondria and fibrillar lattices (or cytoplasmic rays) when analysed by conventional procedures for electron microscopy (Fig. 1A). CGs appeared close to the egg cortex as very dense opaque granules about $300-500 \mu \mathrm{m}$ in diameter. In all cases these granules showed homogeneous dense contents (Fig. 1A). When the oocytes were analysed after PTA staining, CGs appeared as the most positively stained structures in the cytoplasm (Fig. 1B). This characteristic permits easy recording of the number and location of the CGs, and we observed that $83.05 \%$ appeared very close to the egg cortex, linearly arranged along the cell surface. The rest of the granules $(15.00 \%)$ were internalized and $1.95 \%$ were vacuolated and internalized.

Oocytes collected $20 \mathrm{~h}$ after ovulation showed several changes in their structure. Microvilli and fibrillar lattices appeared clearly diminished (Fig. 1C). CGs showed modifications and some of them appeared vacuolated and/or internalized (Fig. $1 C, D$ ). In this case $38.40 \%$ of the CGs were normal, $24.31 \%$ were vacuolized and $37.29 \%$ internalized. A few vacuolized granules appeared in a cortical position and $54.28 \%$ of the internalized granules were vacuolized or swollen (Fig. 1C, D). Dense structures which could represent swollen CGs or remnants of these structures were common in these oocytes. On the other hand, vesicles with variable content, similar to the lysosome-like vesicles described in aged mouse oocytes (Szollosi, 1971), were also observed (Fig. 1C, D).

Modified oocytes from old females were selected by their morphological characteristics (see Material and Methods) (Fig. 2A). These gametes presented a very irregular surface when observed under light microscopy (Fig. 2A). Electron microscopy of semithin sections of these oocytes stained by the PTA procedure revealed that their surface was modified and that they did not show microvilli like normal gametes (Fig. 2B). CGs usually appeared as clusters of granules in some areas of the egg cytoplasm, whereas other regions were completely devoid of granules. In addition, a few normal granules were close to the cortex and others appeared internalized. In these samples CGs showed different diameters and some of them were vacuolated or swelling. In the regions of the cytoplasm that contained CGs, numerous vesicles containing dense bodies were frequently observed. It was not possible to analyse the percentages of normal, vacuolated or swelling CGs and lysosomal-like vesicles in these oocytes because these structures were not as easily characterized as in the oocytes recovered $20 \mathrm{~h}$ after ovulation (Fig. 2C). Furthermore, these oocytes frequently showed cytoplasmic extrusions which appeared free in the perivitelline space (Fig. 2B). These extrusions showed CGs as well as dense structures and vacuoles with the characteristics described above (Fig. 3).

\section{Discussion}

Ageing is a normal phenomenon which affects different biological parameters (Talbert, 1968). In the mammalian female ageing induce gradual changes that result first in a decrease in fertility and then the end of fertile life (Adams, 1984). Numerous analyses have been undertaken on the changes that occur in both preovulatory and postovulatory aged oocytes, and there is now little doubt that ageing has a deleterious effect on the morphology and physiology (i.e. fertilizing capacity) of the female gamete (Goud et al., 1999; Tarín et al., 2000; Key \& Grifo, 2001). Furthermore, several problems occur if an embryo is originated from these aged oocytes (Chian et al., 1992; Ben-Rafael et al., 1986; Wilcox et al., 1998; Tarín et al., 1999). 

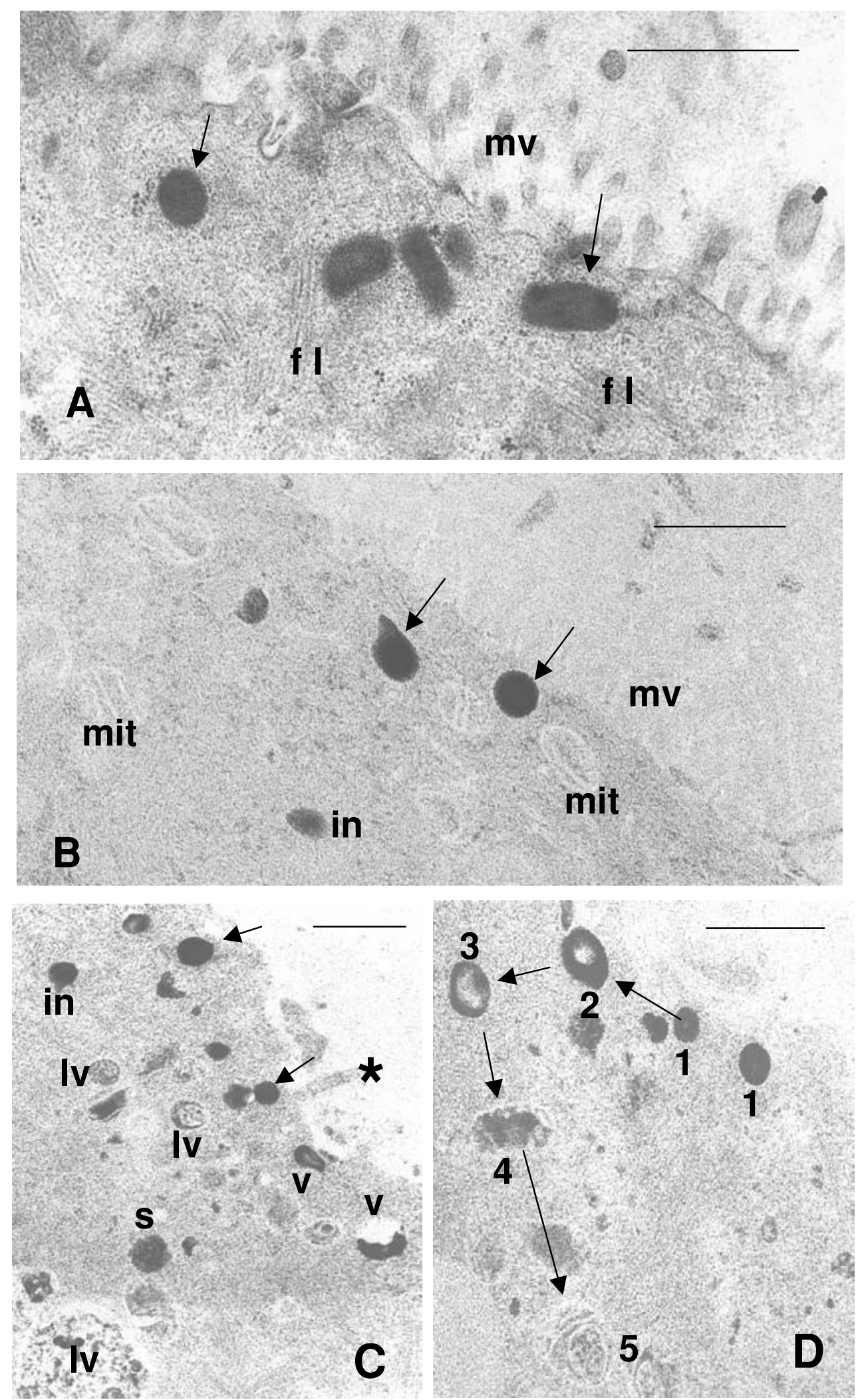

Figure 1 For caption see facing page. 
Analysis of in vitro fertilization of both preovulatory and postovulatory aged oocytes resulted in failures at different steps of fertilization and pronuclear formation (Parkening et al., 1978; Smith \& Lodge, 1987). This factor has also been analysed in humans, showing that numerous changes occur in the nucleus and cytoplasm of the aged oocyte (Lim \& Tsakok, 1997; Krey \& Grifo, 2001). The results we achieved after employing the in vitro fertilization procedure on aged (preovulatory and postovulatory) oocytes are similar to those reported by others, in which a decreased fertility was observed (Winston et al., 1993; Tarín et al., 2000; Goud et al., 1999). Furthermore, physiological changes have been described in the CGs of in vivo aged oocytes, because they were unable to undergo a normal exocytosis when the cortical reaction was in vitro stimulated with the calcium ionophore A231187 (Ducibella et al., 1990). The low rate of fertility of ageing oocytes is not only due to the changes observed in CGs or the egg cortex, and is probably also the result of the numerous modifications that occur in different components of these oocytes, which includes the chromosomes, different cytoplasmic components, the plasma membrane and egg coverings such as the zona pellucida (Szollosi, 1971; Longo, 1974; Tarín et al., 2000, 2001).

CGs are strongly stained by the PTA procedure. This procedure was first used to stain basic proteins (Sheridan \& Barnett, 1969) and later its capacity to selectively contrast lysine-rich basic proteins was identified (Courtens \& Loir, 1981). PTA particularly stains the chromatin, the nucleolus and different structures of invertebrate and vertebrate spermatids and spermatozoa (Holt, 1979; Courtens \& Loir, 1981; Guerra \& Esponda, 1999). The positive PTA staining of the CGs is obviously due to their protein content (Hoodbhoy \& Talbot, 1994). Furthermore, this staining particularity is useful in the analysis of CG distribution and number, as we have shown here. Using this procedure an easy analysis of CGs can be achieved without serial sections in large areas of the oocyte cytoplasm.

Studies on aged mammalian eggs have been done principally in gametes aged in the oviduct after ovulation, and the analysis of oocytes collected from old females has rarely been carried out (Tarín et al., 2000, 2001). The comparison of CGs in freshly ovulated eggs and aged oocytes clearly shows that in the mouse CGs are seriously affected by ageing. During postovulatory ageing CGs are vacuolized, swollen and internalized, and moreover dense structures and lysosome-like vesicles appear. In other cases of postovulatory ageing similar changes have been described: mouse oocytes collected from the oviduct $24 \mathrm{~h}$ after ovulation showed that many CGs had become swollen and internalized (Szollosi, 1971, 1975). Furthermore, in human oocytes aged in culture, CGs also showed a centripetal migration (Sathananthan, 1997). In other species ageing had the opposite effect and CGs moved peripherally, as in the hamster in which CGs may be extruded into the perivitelline space (Yanagimachi \& Chang, 1961), or the rabbit in which postovulatory ageing produce numerous groups of CGs that appear in close contact with the oolemma (Longo, 1985). There is no obvious reason for these species differences in the positioning of CGs during postovulatory ageing, but probably differential changes in the cytoskeleton are responsible for the movements of these physiologically inactive granules. Internalization of CGs is probably produced by changes in the cortical cytoskeleton. During oocyte maturation CGs move peripherally due to microfilaments (Longo, 1985; Sun et al., 2001) and in some species it has been suggested that CG exocytosis is mediated by microfilaments (DiMaggio et al., 1997). In this regard studies have shown that postovulatory ageing produces major alterations in the microfilamentrich area of the egg cortex (Webb et al., 1986; Kim et al., 1996).

The fate of the CG material during oviductal ageing is not known. In Fig. $1 D$ we show a possible sequence of events that could occur: the cortical dense granules would give rise to vacuolated and internalized granules. Then, these internalized granules could change, producing swollen granules or, by their coalescence, dense structures. Lastly, these dense structures would be phagocytosed, resulting in lysosome-like vesicles. These vesicles would represent autophagic vacuoles and have been

Figure $1(A)$ Electron micrograph of the cortical region of a oocyte from a young female recovered soon after ovulation. CGs (arrows) appears as very dense structures close to the plasma membrane. Numerous microvilli (mv) and fibrillar lattices (fl) are indicated. Glutaraldehyde-osmium fixation and uranyl lead staining. $(B)$ An oocyte similar to that in $(A)$ treated with the PTA method. CGs (arrows) are observed as the most stained structures in the cytoplasm, and some of them are internalized (in). Microvilli (mv) and mitochondria (mit) can be seen. (C) Cortical cytoplasm of a oocyte recovered from a young female $20 \mathrm{~h}$ after ovulation. PTA staining. Some CGs show normal characteristics (arrows), while others are vacuolated (v), internalized (in) or swollen (s). Lysosome-like vesicles (lv) are also observed. Microvilli $\left(^{*}\right)$ are scarce in these oocytes. $(D)$ A similar oocyte in which the arrows show the possible evolution of CGs: normal CGs (1) would form vacuolated granules (2), some of which are internalized (3). These granules could be the origin of swollen granules (4) and of lysosomal-like vesicles (5). Scale bars represent $0.1 \mu \mathrm{m}$. 


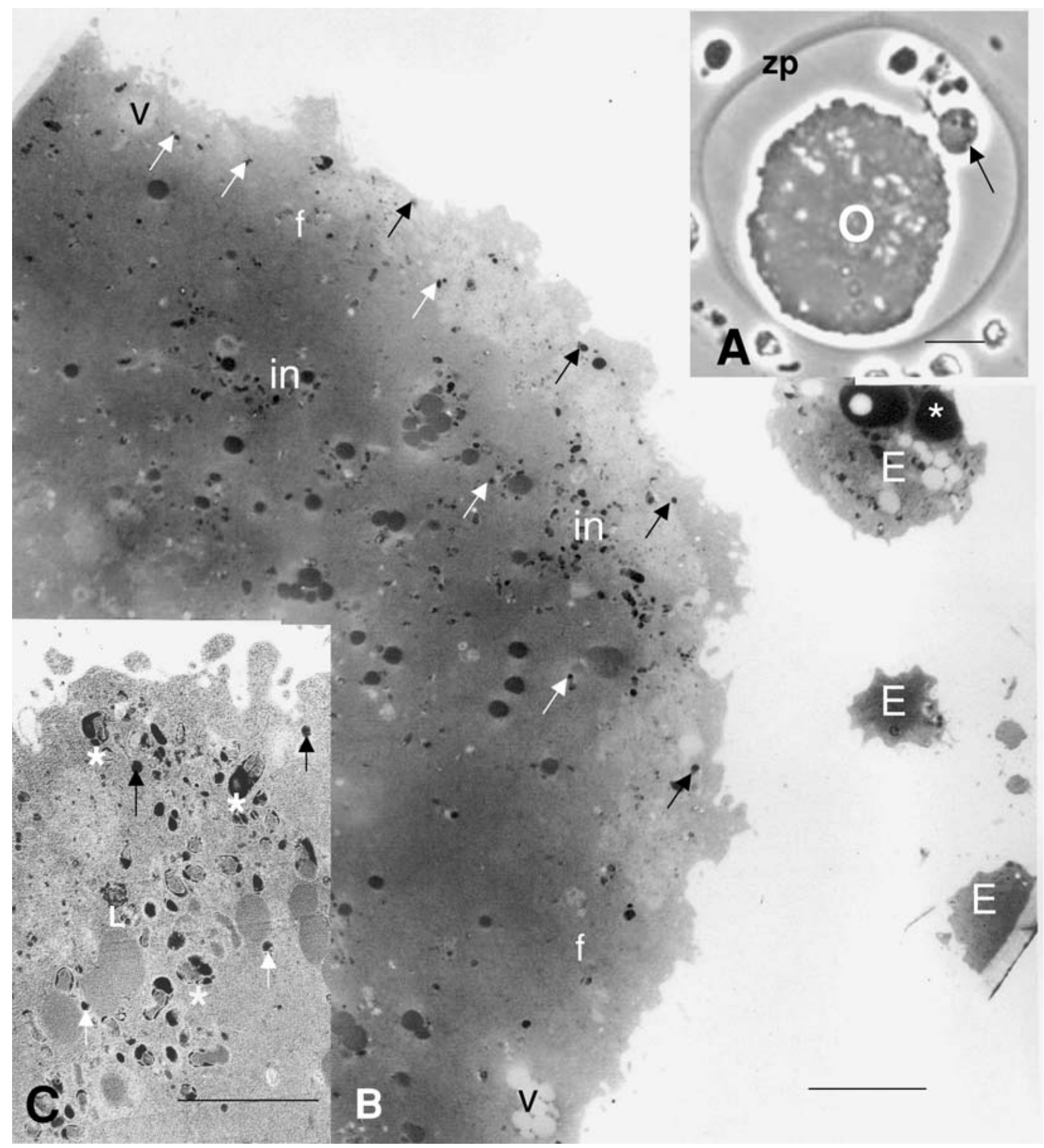

Figure 2 Oocytes recovered from old females stained with PTA. $(A)$ Semithin section of an oocyte $(\mathrm{O})$ stained with toluidine blue and observed under phase-contrast microscopy. There is a broad perivitelline space in which numerous cytoplasmic extrusions occur (arrow). Zp, zona pellucida. (B) Section $0.5 \mu \mathrm{m}$ thick shows part of the cortical region of the oocyte observed in $(A)$. The surface of the oocyte is irregular and does not show microvilli. In the cortex there are areas with numerous CGs and others free of granules (f); in addition groups of internalized granules are observed (in). Some normal CGs appears close to the oolemma (black arrows) and others are internalized (white arrows). In the perivitelline space, cytoplasmic extrusions (E) show CGs and large dense structures $\left(^{*}\right) .(C)$ Part of the cortex showing an area rich in granules. The irregular surface of the oocyte is observed. Some normal CGs appears close to the cortex (black arrows) or internalized (white arrows). Numerous dense structures appear in these regions of the cortex, some of which are large $\left(^{*}\right)$ while others resemble lysosome-like vesicles (L). Scale bars represent: $(A) 20 \mu \mathrm{m} ;(B): 2 \mu \mathrm{m} ;(C): 0.5 \mu \mathrm{m}$. 


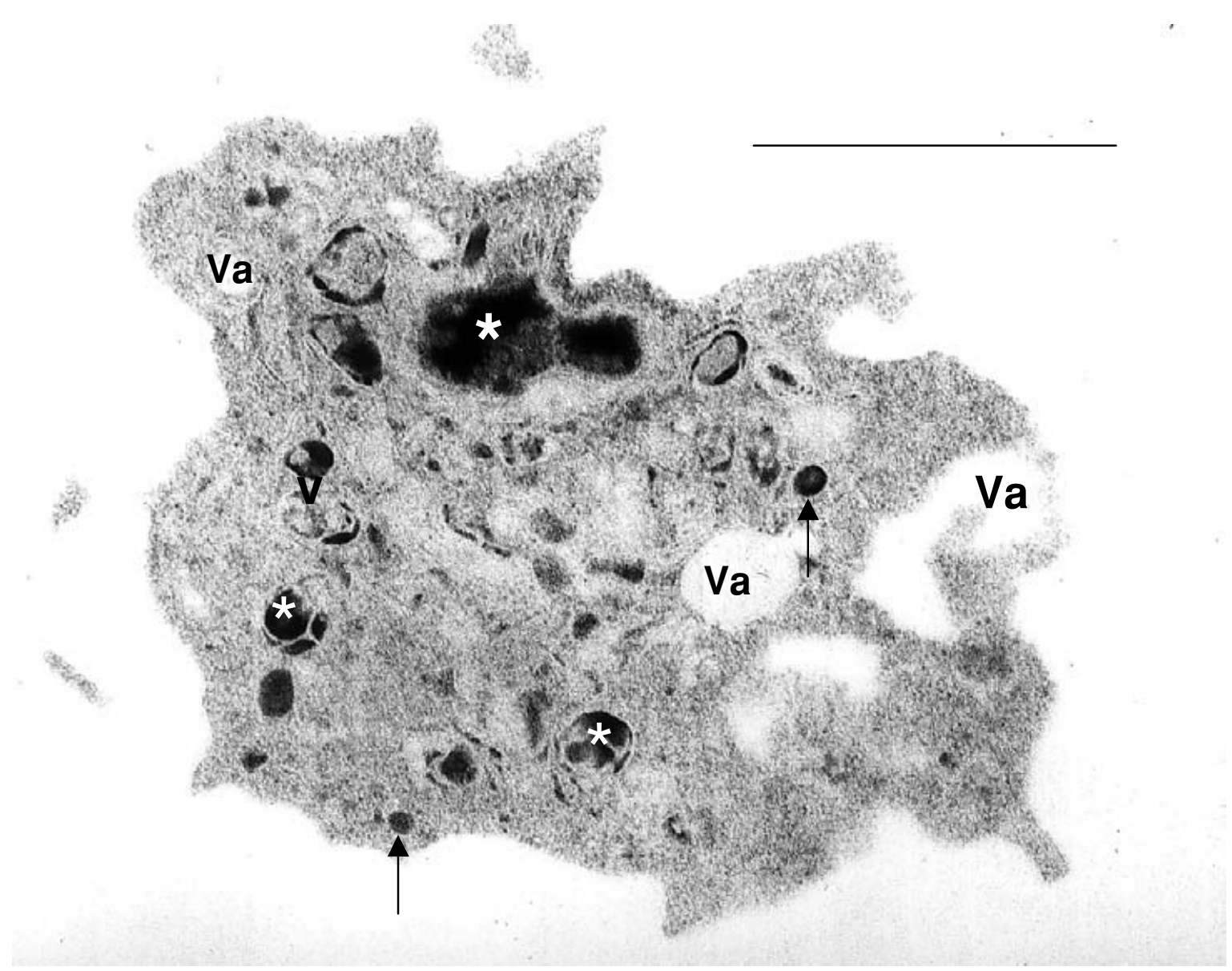

Figure 3 Cytoplasmic extrusion in an oocyte ovulated by an old female. PTA staining. Normal CGs (arrows), vacuoles (va) and numerous large dense structures $\left(^{*}\right)$ are observed. Scale bar represents $0.5 \mu \mathrm{m}$.

demonstrated previously in mouse aged oocytes (Szollosi, 1971).

The ultrastructural picture in oocytes collected from old females is different and more complicated, and CG internalization seems to be the predominant alteration. In these oocytes some areas of cytoplasm appeared free of granules, a fact that would indicate cytoplasmic reorganization had occurred. On the other hand, numerous vacuolated dense structures appeared. The origin of these structures is unknown but they could represent the remnants of swollen CGs, lysosome-like vesicles or remnants of other cytoplasmic structures. All these changes are undoubtedly related to the degenerative changes occurring in these oocytes. Analysis of the production of degenerated oocytes by aged female mice has shown that the percentage of degenerated oocytes increases with age and that females from 50 to 62 weeks old produced large numbers of anomalous or degenerated oocytes (Tarín et al., 2001). These degenerated gametes constitute at least a proportion of the gametes that are not fertilizable when in vitro fertilization procedures are used (Lim \& Tsakok, 1997).
Ultrastructural analysis showed that ageing produces modifications in other structures of the oocyte. The oolemma changes rapidly after ovulation and the loss of microvilli is evident, a phenomenon previously shown in preovulatory aged oocytes (Peluso et al., 1980). In the rabbit striking modifications were observed in oocytes aged in vivo, in which the perivitelline space appeared full of blebs formed by the oocyte surface (Longo, 1974). Structures such as fibrillar lattices, which are characteristic of rodent oocytes and would serve as a storage material (Gosden \& Bownes, 1995), show a clear deterioration after preovulatory and postovulatory ageing, a fact that was previously shown in oocytes collected from old rats (Peluso et al., 1980).

Cytoplasmic fragments have been usually noted in aged oocytes (Szollosi, 1971; Longo, 1974; Tarín et al., 2001) and are also common in cultured mammalian embryos (Xu et al., 2001). Our observations showed that these cytoplasmic fragments have similar morphological characteristics to the oocyte cortical cytoplasm, containing normal and modified CGs, vacuoles and dense structures. Generally speaking, cytoplasmic fragmentation is the visual evidence of 
a degenerative change in gametes or embryos. In the mouse, oocytes recovered $18-24 \mathrm{~h}$ after ovulation showed fragmentation (Szollosi, 1971), a fact that was also observed in the rabbit (Longo, 1974) and the rat, where they appear in gametes collected from old females (Peluso et al., 1980). Moreover the ultrastructural analysis of fragments has shown that fragmentation involves the presence of cytoplasmic constrictions similar to those formed after cell cleavage, indicating that this is a phenomenon that resembles cytokinesis (Szollosi, 1971). On the other hand, cytoplasmic fragmentation could be also related to the apoptotic phenomenon that has been described in aged oocytes (Fujino et al., 1996; Pérez et al., 1999).

In conclusion, remarkable changes appeared in the mouse CGs and in the egg cortex after postovulatory and preovulatory ageing. Postovulatory changes occur in a short time $(20 \mathrm{~h})$, and the main change is internalization of CGs, which indicates the oocyte's inability to develop a normal cortical reaction. In addition, vacuolation, swelling and other changes appeared as a consequence of this quick ageing in the oviductal environment, which seems to be rapidly deleterious for the oocyte. Changes occurring after preovulatory ageing are closely related to degenerative changes (i.e. formation of dense pyknotic structures, cytoplasmic fragmentation, etc.). All these modifications are obviously related to the low fertility of these gametes.

\section{Acknowledgements}

This work was supported by Grant BCM2000-0899 from CICYT (Spain). H.D. is grateful for support from AECI (Spain). The authors wish to thank Ascensión González and Elena Rodriguez for their valuable technical help.

\section{References}

Adams, C.E. (1984). Reproductive senescence. In Reproduction in Mammals, vol. 4, Reproductive Fitness (ed. C.R. Austin \& R.V. Short), pp. 210-33. Cambridge: Cambridge University Press.

Ben-Rafael, Z., Kopf, G.S., Blasco, L., Tureck, R.W. \& Mastroianni, L. (1986). Fertilization and cleavage alter reinsemination of human oocytes in vitro. Fertil. Steril. 45, 58-62.

Chian, R.C., Nakahana, H., Niwa, K. \& Funahashi, H. (1992). Fertilization and early cleavage in vitro of ageing bovine oocytes after maturation in culture. Theriogenology 37, 66572.

Courtens, J.L. \& Loir, M. (1981). Ultrastructural detection of basic nucleoproteins: alcoholic phosphotungstic acid does not bind arginine residues. J. Ultrastruct. Res. 74, 322-6.
Dimaggio, A.J., Lonergan, T.A. \& Stewart-Savage, J. (1997) Cortical granule exocytosis in hamster eggs requires microfilaments. Mol. Reprod. Dev. 47, 334-40.

Ducibella, T.T. (1996). The cortical reaction and development of activation competence in mammalian oocytes. Hum. Reprod. Update 2, 29-42.

Ducibella, T., Duffy, P., Reindollar, R, \& Su, B. (1990). Changes in the distribution of mouse oocyte cortical granules and ability to undergo the cortical reaction during gonadotropin-stimulated meiotic maturation and aging in vivo. Biol. Reprod. 43, 870-6.

Fujino, Y., Ozaki, K., Yamamasu, S., Ito, F., Matsuoka, I., Hayasi, E., Nakamura, H., Ogita, S., Sato, E. \& Inoue, M. (1996). DNA fragmentation of oocytes in aged mice. Hum. Reprod. 11, 1480-3.

Gosden, R.G. \& Bownes, M. (1995). Molecular and cellular aspects of oocyte development. In Gametes. The Oocyte (ed. J.G. Grudzinskas \& J.L. Yovich), pp. 23-53. Cambridge: Cambridge University Press.

Goud, P., Goud, A., Van Oostveldt, O., Van der Elst, J. \& Dhont, M. (1999). Fertilization abnormalities and pronucleus size asynchrony after intracytoplasmic sperm injection are related to oocyte postmaturity. Fertil. Steril. 72, 245-52.

Guerra, R. \& Esponda, P. (1999). Structure, cytoskeleton and development of the acrosome of Platycleis albopunctata (Orthoptera: Tettigoniidae). J. Morphol. 242, 47-56.

Gulyas, B.J. (1980). Cortical granules of mammalian eggs. Int. Rev. Cytol. 63, 357-92.

Guraya, S.S. (1982). Recent progress in the structure, origin, composition and function of cortical granules in animal eggs. Int. Rev. Cytol. 78, 257-360.

Holt, W.V. (1979). Development and maturation of the mammalian acrosome. A cytochemical study using phosphotungstic acid staining. J. Ultrastruct. Res. 68, 58-71.

Hoodbhoy, T. \& Talbot, P. (1994). Mammalian cortical granules: contents, fate, and function. Mol. Reprod. Dev. 39, 439-48.

Kim, N.Y., Moon, S.J., Prather, R.S. \& Day, B.N. (1996). Cytoskeletal alteration in aged porcine oocytes and parthenogenesis. Mol. Reprod. Dev. 43, 513-18.

Krey, L.C. \& Grifo, J.A. (2001). Poor embryo quality: the answer lies (mostly) in the egg. Fertil. Steril. 75, 466-8.

Lim, A.S.T. \& Tsakok, M.F.H. (1997). Age-related decline in fertility: a link to degenerative oocytes. Fertil. Steril. 68, 265-71.

Longo, F.J. (1974). Ultrastructural changes in rabbit eggs aged in vivo. Biol. Reprod. 11, 22-39.

Longo, F.J. (1985). Fine structure of mammalian egg cortex. Am. J. Anat 174, 303-15.

Parkening, T.A., Lau, I.F., Saksena, S.K. \& Chang, M.C. (1978). Circulating plasma levels of pregnenolone, progesterone, estrogen, luteinizing hormone, and follicle stimulating hormone in young and aged C57BL/ 6 mice during various stages of pregnancy. J. Gerontol. 33, 191-6.

Peluso, J.J., England-Charlesworth, C. \& Hutz, R. (1980). Effect of age and of follicular aging on the preovulatory oocyte. Biol. Reprod. 22, 999-1005.

Pérez, G.L., Tao, X. \& Tilly, J.L. (1999). Fragmentation and death of ovulated oocytes. Mol. Hum. Reprod. 5, 41420. 
Sathananthan, A.H. (1997). Ultrastructure of the human egg. Hum. Cell 10, 21-38.

Sheridan, W.F. \& Barnett, R.J. (1969). Cytochemical studies on chromosome ultrastructure. J. Ultrastruct. Res. 27, 216-29.

Smith, A.L. \& Lodge, J.R. (1987). Interactions of aged gametes: in vitro fertilization using in vitro-aged sperm and in vivoaged ova. Gamete Res. 16, 47-56.

Sun, Q.Y., Lai, L., Park, K.W., Kühholzer, B., Prather, R.S. \& Schatten, H. (2001). Dynamic events are differently mediated by microfilaments, microtubules, and mitogenactivated protein kinase during porcine oocyte maturation and fertilization in vitro. Biol. Reprod. 64, 871-89.

Szollosi, D. (1971). Morphological changes in mouse eggs due to aging in the Fallopian tube. Am. J. Anat. 130, 209-26.

Szollosi, D. (1975). Mammalian eggs aging in the fallopian tubes. In Aging Gametes. Their Biology and Pathology (ed. R.J. Blandau), pp. 98-121. Basel: Karger.

Talbert, G.B. (1968). Effect of maternal age on reproductive capacity. Am. J. Obstet. Gynecol. 102, 451-77.

Tarín, J.J., Pérez-Albalá, S., Aguilar, A., Miñarro, J., Hermenegildo, C. \& Cano, A. (1999). Long-term effects of postovulatory aging of mouse oocytes on offspring: a two-generational study. Biol. Reprod. 61, 1347-55.

Tarín, J.J., Pérez-Albalá, S. \& Cano, A. (2000). Consequences on offspring of abnormal function in ageing gametes. Hum. Reprod. Update 6, 532-49.

Tarín, J.J., Perez Albalá, S. \& Cano, A. (2001). Cellular and morphological traits of oocytes retrieved from aging mice after exogenous ovarian stimulation. Biol. Reprod. 65, 14150.

Webb, M., Howlett, S.K. \& Maro, B. (1986). Parthenogenesis and cytoskeletal organization in ageing mouse eggs. J. Embryol. Exp. Morphol. 95, 131-45.

Wilcox, A.J., Weinberg, C.R. \& Baird, D. (1998). Post-ovulatory ageing of human oocyte and embryo failure. Hum. Reprod. 13, 394-7.

Winston, N.J., Braude, P.R. \& Johnson, M.H. (1993). Are failed-fertilized human oocytes useful? Hum. Reprod. 8, 503-7.

Xu, J., Cheung, T., Chan, S.T., Ho, P. \& Yeung, W.S. (2001). The incidence of cytoplasmic fragmentation in mouse embryos in vitro is not affected by inhibition of caspase activity. Fertil. Steril. 75, 986-91.

Xu, Z., Abbott, A., Kopf, G.S., Schultz, R.M. \& Ducibella, T. (1997). Spontaneous activation of ovulated eggs: time dependent effects on M-phase exit, cortical granule exocytosis, maternal messenger ribonucleic acid recruitment, and inositol 1,4,5-triphosphate sensitivity. Biol. Reprod. 57, 743-50.

Yanagimachi, R. (1988). Mammalian fertilization. In The Physiology of Reproduction, vol. 1 (ed. E. Knobil \& J.D. Neil), pp. 135-85. New York: Raven Press.

Yanagimachi, R. \& Chang, M.C. (1961). Fertilizable life of golden hamster ova and their morphological changes at the time of losing fertilizability. J. Exp. Zool. 148, 185204. 
